\title{
Gafanhotos (Orthoptera, Acridoidea) em áreas de cerrados e lavouras na Chapada dos Parecis, Estado de Mato Grosso, Brasil
}

\author{
Wanderlei Dias Guerraํㄹ Patrícia Carla de Oliveira² \& José Roberto Pujol-Luz ${ }^{3}$
}

\begin{abstract}
'Programa de Pós-graduação em Biologia Animal, ${ }^{3}$ Departamento de Zoologia, Instituto de Ciências Biológicas, Universidade de Brasília, UnB, 70.010-900 Brasília-DF, Brasil. wanderlei.dias@agricultura.gov.br

${ }^{2}$ Departamento de Botânica e Ecologia, Instituto de Biociências, Universidade Federal de Mato Grosso, UFMT, 78.060-900 Cuiabá-MT, Brasil. patiranjak@yahoo.com.br

${ }^{3}$ Departamento de Zoologia, Instituto de Ciências Biológicas, Universidade de Brasília, UnB, 70.010-900 Brasília-DF, Brasil. jrpujol@unb.br
\end{abstract}

\begin{abstract}
Grasshoppers (Orthoptera, Acridoidea) in native savanna and crop areas in Chapada dos Parecis, Mato Grosso State, Brazil. We determined the composition and abundance of grasshoppers using sweep net sampling during three years at the Parecis Plateau, State of Mato Grosso, Brazil. The survey was done in areas with crops and native vegetation (savanna) with, respectively, 56 and 59 sites available in each environment. 3.031 individuals of grasshoppers were collected from 64 species distributed among the following families and subfamilies: Acrididae (49): Gomphocerinae (21), Ommatolampinae (10), Melanoplinae (6), Acridinae (4) Leptysminae (3), Copiocerinae (3), Proctolabinae (1) and Cyrtacanthacridinae (1); Romaleidae (1): Romaleinae (13) and Ommexechidae (1): Ommexechinae (2) and 1550 nymphs. The differences were significant between the number of species found in the savannas (61) and in cultived areas (16). However, the abundance of Acridoidea was significantly higher in crops than in savannas areas which was influenced mainly by two Acrididae's species: Baeacris punctulatus (Thunberg, 1824) and Orphulella punctata (De Geer, 1773) which accounted for $49.5 \%$ of the total individuals collected across the Parecis Plateau and, together, they account for $78.8 \%$ of recorded abundance in the cultived areas, both species being potential pests.
\end{abstract}

KEYWORDS. Brazilian savanna; community structure; composition; Melanoplinae; pest; richness.

RESUMO. Gafanhotos (Orthoptera, Acridoidea) em áreas de cerrados e lavouras na Chapada dos Parecis, Estado de Mato Grosso, Brasil. Foi determinada a composição e abundância de espécies de gafanhotos usando amostragem com rede entomológica durante 3 anos de estudo na Chapada dos Parecis, estado de Mato Grosso. O levantamento foi feito em áreas de lavouras e com vegetação ainda nativa (cerrados) com, respectivamente, 56 e 59 locais inventariados em cada ambiente. Foram coletados 3.031 indivíduos de gafanhotos de 64 espécies distribuídas entre as famílias e subfamílias: Acrididae (49): Gomphocerinae (21), Ommatolampinae (10), Melanoplinae (6), Acridinae (4) Leptysminae (3), Copiocerinae (3), Proctolabinae (1) e Cyrtacanthacridinae (1); Romaleidae (1): Romaleinae (13) e Ommexechidae (1): Ommexechinae (2), além de 1550 ninfas. A diversidade de espécies foi maior no cerrado (61) do que nas lavouras (16), ocorrendo o inverso com relação à abundância onde as espécies Baeacris punctulatus (Thunberg, 1824) e Orphulella punctata (De Geer, 1773) predominaram representando 49,5\% do total de indivíduos coletados em toda a Chapada dos Parecis e, juntas, somam $78,8 \%$ da abundância registrada nas áreas de lavouras e tem potencial de se tornarem pragas.

PALAVRAS-CHAVE. Cerrado; composição; estrutura de comunidade; Melanoplinae; praga; riqueza.

O Cerrado, bioma característico do Planalto Central, com grande diversidade de flora e de fauna, foi incluído na lista dos hotspots em diversidade do planeta pela Conservation Internacional (Mittermeier et al. 1999) devido a ameaças que vem sofrendo em virtude da ação do homem. As diminuições territoriais deste bioma ocorridas ao longo dos anos, em especial a partir da década de 1980 , tem se tornado uma preocupação da comunidade científica.

Vários trabalhos vem sendo desenvolvidos visando o conhecimento da entomofauna da região de Cerrado (Santos et al. 2004; Constantino 2005; Soares et al. 2010), mas poucos avanços ocorreram em relação ao conhecimento da ortopterofauna, principalmente no que se trata de Planalto Central e especialmente no estado de Mato Grosso, um dos grandes responsáveis pelas alterações deste bioma.

Os gafanhotos constituem um dos maiores, e possivelmente o mais dominante, grupo de insetos herbívoros da Terra
(Gangwere et al. 1997), características que os tornam insetos especialmente importantes para o sistema de defesa fitossanitária visando à proteção das plantas cultivadas. Apesar de 95\% das espécies de gafanhotos não serem pragas, aquelas que são causam danos econômicos expressivos (Gangwere et al. 1997).

No Brasil são relatadas pelo menos 20 espécies de gafanhotos com importância econômica ocorrendo em diferentes regiões geográficas (Lecoq 1991; Guerra 2001). Recentemente, Baeacris punctulatus Thunberg, 1824 foi relatada causando danos em lavouras mato-grossenses, em especial no município de Campo Novo do Parecis (Guerra et al. 2010). Esta espécie e outras sobrevivem durante o inverno (período de entressafra) em meio aos cultivos de milheto, sorgo, crotalária e girassol, culturas que depois de colhidas ou dessecadas dão lugar às lavouras de soja, passando esta a ser a alternativa de alimentação. 
Os ambientes de cerrado possuem estrutura de habitat completamente distintas das lavouras. Sua composição fitossociológica apresenta formações florestais, savânicas e campestres, nas quais varia a dominância de árvores, arbustos, subarbustos e ervas, em especial as gramíneas. Tal variabilidade de composições cria condições ecológicas antagônicas às das lavouras, cujas paisagens são muito homogêneas. A grande diversidade de espécies vegetais que caracteriza os habitats do cerrado influencia na estruturação das taxocenoses de gafanhotos destes ambientes, assim como ocorre em outros biomas como as savanas africanas, cuja composição florística determina a composição das espécies de gafanhotos (Gebeyehu \& Samways 2002).

A análise comparativa da composição de gafanhotos dos dois ambientes é importante para revelar a presença de pragas em potencial e os resultados obtidos podem orientar ações preventivas à sua ocorrência e estudos futuros visando o monitoramento de alterações nas paisagens, além de subsidiar as políticas públicas.

Lecoq (1991) alertou que o homem pode, ao modificar o ambiente, favorecer o gafanhoto propiciando novos meios a certas espécies que antes eram inofensivas. De acordo com diferentes autores, as explosões populacionais de gafanhotos em certos estados do Brasil (Rondônia e Mato Grosso, especialmente) estão certamente ligadas, em grande parte, ao desmatamento, ocasionando desequilíbrio ecológico e significativa redução dos inimigos naturais, favorecendo sua explosão populacional (Carbonell 1988; Lecoq 1991). Da mes- ma forma os novos cultivos introduzidos nas áreas recém abertas certamente dão uma grande vantagem competitiva para um subconjunto dos gafanhotos, influenciando na sua capacidade de sobrevivência e o tamanho populacional (Begon et al. 2006).

Este trabalho tem como objetivo descrever a composição da comunidade de gafanhotos da Chapada dos Parecis, Mato Grosso, bem como avaliar as diferenças dessa composição entre os dois ambientes predominantes na região, vegetação de cerrado e lavouras, através da análise da abundância, riqueza e estrutura da taxocenose de gafanhotos.

\section{MATERIAL E MÉTODOS}

Este estudo foi conduzido entre os anos de 2008 e 2010 em propriedades agrícolas e áreas preservadas situadas na unidade geomorfológica da Chapada dos Parecis, estado de Mato Grosso, dentro dos limites dos municípios de Sapezal, Campo Novo do Parecis, Tangará da Serra e Campos de Júlio (Fig. 1). A área está delimitada entre os paralelos $13^{\circ} 30^{\prime}$ a $14^{\circ} 45^{\prime} \mathrm{S}$ e $57^{\circ} 00^{\prime}$ a $59^{\circ} 30^{\prime} \mathrm{W}$, compreendendo aproximadamente $20.000 \mathrm{~km}^{2}$. Sobre esta unidade, originalmente de domínio biogeográfico de Cerrado, estão instalados grandes e recentes empreendimentos agrícolas. A região se caracteriza por um planalto de altitude, com elevações entre 550 e 800 m, solos tipo Areias Quartzosas (Neossolo Quartzarênico) e Latossolos, predomínio de vegetação de Cerrados e clima tropical com inverno seco e verão úmido e temperaturas médias variando entre 20 e $25^{\circ} \mathrm{C}$ (Brasil 1982).

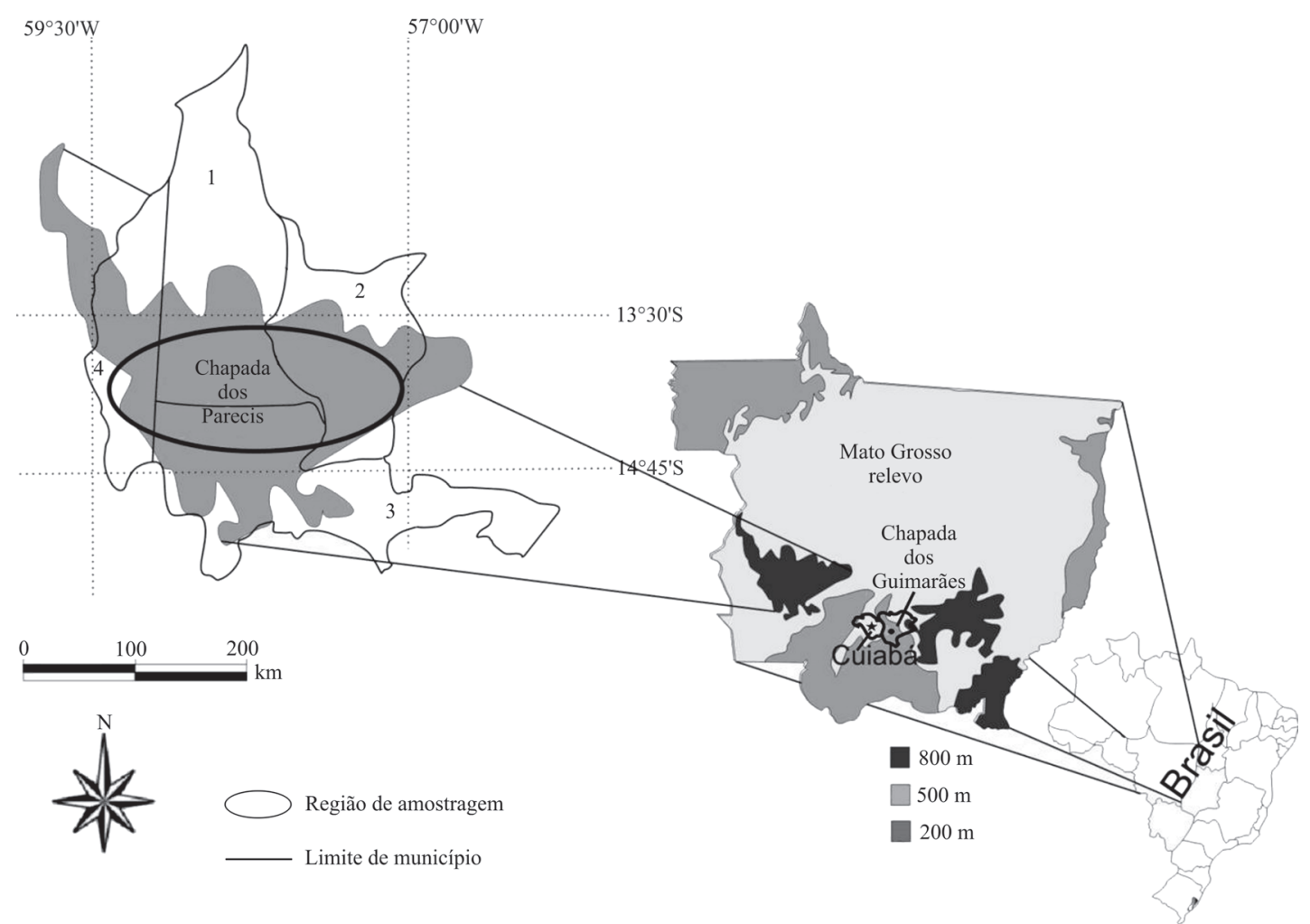

Fig. 1. Localização da região amostrada na Chapada dos Parecis, Mato Grosso, Brasil. Municípios: 1: Sapezal; 2: Campo Novo do Parecis; 3: Tangará da Serra; 4: Campos de Júlio. Adaptado de: http://www.ibge.gov.br/mapas_ibge. 
A amostragem contemplou dois tipos de ambientes, que respondem pela maior parte da cobertura da Chapada dos Parecis. O primeiro, aqui referido como ambiente antropizado (ou lavouras), compreende as áreas de agricultura intensiva, sendo a expressão mais comum o cultivo de soja sucedido por culturas de entressafra, tais como milho, algodão, girassol, milheto e crotalária. O segundo, denominado ambiente de cerrados (ou área nativa), levou em conta as fitofisionomias de cerrado sentido restrito e formações campestres (Ribeiro \& Walter 1998), devido ao predomínio de tais fitofisionomias na região.

Foram amostrados 115 pontos, sendo 59 no ambiente de cerrados e 56 no ambiente de lavouras, distribuídos segundo a predominância destes ambientes e suas subdivisões na área de estudo, e buscando o alcance de toda a Chapada. A coleta foi feita pela combinação de dois métodos diferentes de ampla aceitação para este tipo de estudo (Duranton et al. 1982), que aliou o uso de rede entomológica e a busca um a um dos Acridoidea que pudessem ser encontrados na área amostrada.

Em cada ponto de amostragem foi delimitada uma área de $50 \mathrm{~m} \times 50 \mathrm{~m}$, dividida em quatro sub-parcelas para facilitar a busca dos gafanhotos. Em cada uma das quatro sub-parcelas foram aplicados 50 movimentos de coleta com rede entomológica, incluindo os topos das plantas arbustivas, totalizando 200 golpes por ponto de amostragem (adaptado de Wysiecki et al. 2005). Para a busca ativa dos insetos foi estabelecido um tempo de 60 minutos por parcela de $50 \times 50 \mathrm{~m}$, o qual foi definido a partir de ensaio prévio e obtenção da curva do coletor.

As amostragens contemplaram os períodos secos e úmidos ao longo do ano, buscando com isto o maior alcance da composição dos Acridoidea. Também foi considerada a distribuição equilibrada dos horários de coleta entre os dois ambientes, eliminando assim uma possível fonte de erro nas análises comparativas.

Os insetos coletados foram acondicionados em recipientes contendo acetato de etila, com posterior separação dos adultos e ninfas, quantificação e identificação das espécies. Para preservar a coloração original os adultos foram eviscerados seguindo a técnica de Rosas-Costa (1966). Para a identificação taxonômica seguiu-se o proposto por Amédégnato (1974), além da comparação com a Coleção de Referência de Gafanhotos Praga da Superintendência Federal de Agricultura em Mato Grosso e do Museu Nacional do Rio de Janeiro, consulta a especialistas e busca no site Orthoptera Species Files-2 (OSF2) (Eades et al. 2009). Parte do material coletado será mantido como coleção de referência do Ministério da Agricultura, Pecuária e Abastecimento - MAPA, junto à Superintendência Federal de Agricultura em Mato Grosso, na Coleção Entomológica do Instituto de Biologia da Universidade de Brasília - UnB e também junto ao Museu Nacional da Universidade Federal do Rio de Janeiro - UFRJ.

A composição dos ortópteros na Chapada dos Parecis é descrita pela lista de espécies obtida, pela riqueza adotada como índice de diversidade e pelo ranqueamento das espécies de acordo com suas abundâncias (Magurran 2004). Tais parâmetros são apresentados comparativamente para os dois ambientes (lavouras e áreas nativas).

Para as análises estatísticas foi usado o programa $\mathrm{R}$ versão 2.11.1 (R Development Core Team 2009). Para detecção de diferenças na distribuição da abundância e riqueza entre cerrados e lavouras foi aplicado teste " $t$ " com comando t.test do pacote vegan (Oksanen et al. 2009), após verificada a normalidade das distribuições. Para detecção das diferenças entre as espécies com maiores variações nas abundâncias entre os dois ambientes amostrados foi aplicado o teste Kruskal-Wallis, utilizando o Programa estatístico PAST (Hammer et al. 2001).

A curva de acumulação de espécies baseada no número de pontos amostrados na Chapada dos Parecis foi gerada utilizando-se o programa estatístico EstimateS versão 8.2.0 (Colwell 2006) e os gráficos e figuras construídos no programa Microsoft Excel versão 1997-2003.

As estimativas de riqueza para os ambientes naturais e antropizados foram feitas usando os estimadores de riqueza não paramétricos Jackknife de segunda ordem e Chao 2, conforme proposto por Gotelli \& Colwell (2001), com intervalo de confiança de $95 \%$. Na construção do ranking de abundâncias, também conhecido como diagrama de dominância ou de Whittaker, as abundâncias das espécies (n) foram transformadas utilizando $\log _{10}(\mathrm{n}+1)$ (Magurran 2004).

\section{RESULTADOS}

Durante os três anos de estudo foram coletados 3.031 indivíduos de gafanhotos adultos, dos quais 1.202 (39,7\%) nos cerrados e $1.829(60,3 \%)$ nas áreas de lavouras. Obtevese 64 espécies, distribuídas entre as seguintes famílias e subfamílias: Acrididae (49): Gomphocerinae (21), Ommatolampinae (10), Melanoplinae (6), Acridinae (4) Leptysminae (3), Copiocerinae (3), Proctolabinae (1) e Cyrtacanthacridinae (1); Romaleidae (13): Romaleinae (13) e Ommexechidae (2): Ommexechinae (2), das quais 61 ocorrendo nos habitats de cerrado e 16 nas lavouras (Tabela I).

A subfamília Melanoplinae foi a mais abundante, representando $37,9 \%$ dos gafanhotos coletados neste estudo e o total de espécimes desta subfamília coletadas nas lavouras representaram $56,2 \%$ de toda a abundância neste ambiente, seguido dos Gomphocerinae com 31,6\% da abundância geral da Chapada dos Parecis. Esta última subfamília teve a maior abundância relativa dentre as coletas feitas nos cerrados, representando $42,4 \%$ de todos os gafanhotos capturados neste ambiente, e também contribuiu com $24,5 \%$ de todos os espécimes encontrados nas lavouras. Gomphocerinae foi a subfamília que teve uma proporção mais equilibrada entre indivíduos coletados nos cerrados $(53,3 \%)$, e nas lavouras $(46,7 \%)$. As demais subfamílias, com exceção da já citada Melanoplinae com $89,6 \%$ de sua abundância amostrada nas lavouras e da Cyrtacanthacridinae com 95,9\%, tiveram seus indivíduos coletados quase que exclusivamente nos cerrados (Tabela I). Todas as 13 espécies de Romaleidae foram encontradas apenas nos cerrados. 
Dentre as espécies catalogadas, 48 (75\%) foram exclusivas de ambientes de cerrado e somente 13 (20\%) são de ocorrência comum aos dois ambientes. Foram coletadas 1.550 ninfas, tendo sido identificadas somente as de B. punctulatus. As abundâncias totais de ninfas foram distribuídas com 62,9\% nas lavouras e $37,1 \%$ nos cerrados, equivalendo a $51,2 \%$ do total de adultos coletados (Tabela I).

A riqueza de espécies, também assumida como índice de diversidade, foi significativamente maior no cerrado (61) do que nas áreas antropizadas com lavouras (16) $(\mathrm{t}=8,0 ; \mathrm{p}<$ 0,001) (Fig. 2), o que pode também ser demonstrado pelas curvas de acúmulo de espécies (Fig. 3). As estimativas da riqueza total resultaram em valores mínimos pelo estimador Chao 2: 71 para os cerrados e 25 para as lavouras, e máximos para o estimador Jackknife 2 com 80 espécies nos cerrados e 26 nas lavouras. A riqueza por local amostrado variou de zero a seis em áreas de lavouras e de um a dezesseis nos cerrados. As médias, acompanhadas de seus desvios, observadas nos dois ambientes foram 7,1 $\pm 3,7$ nos cerrados e $2,8 \pm 1,6$ nas lavouras.

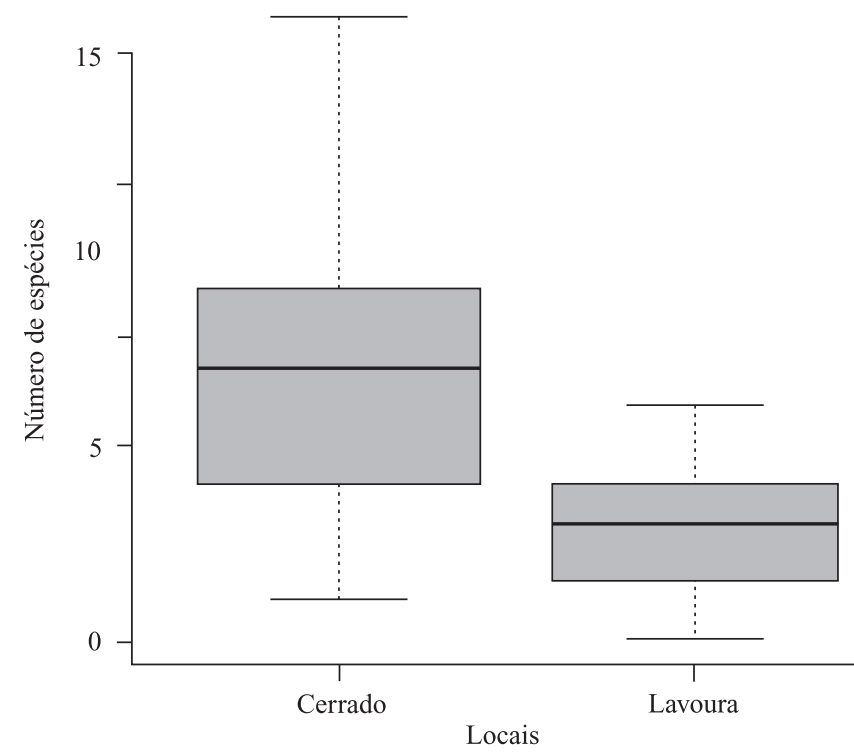

Fig. 2. Riqueza de espécies de gafanhotos observada em 59 pontos nos cerrados (total $=61$ espécies) e 56 nas lavouras (total $=16$ espécies) da Chapada dos Parecis, Mato Grosso. A linha horizontal em negrito é a mediana, as caixas representam os quartis adjacentes à mediana e as hastes pontilhadas correspondem aos valores extremos. Áreas nativas comportam uma riqueza significativamente maior que as áreas de lavouras $(\mathrm{p}<0,001)$.

A abundância de gafanhotos foi significativamente maior nas lavouras que nos cerrados $(\mathrm{t}=-2.14 ; \mathrm{p}=0,035)$ (Fig. 4). As médias por ponto de amostragem, acompanhadas de seus desvios, observadas nos dois ambientes foram $20 \pm 18$ nos cerrados e $33 \pm 39$ nas lavouras.

Apenas duas espécies da família Acrididae, B. punctulatus e Orphulella punctata De Geer, 1773, responderam por 49,5\% do total de indivíduos coletados em toda a Chapada dos Parecis e, juntas, somam $78,8 \%$ da abundância registrada nas áreas de lavouras. A espécie $B$. punctulatus respondeu sozinha por $35 \%$ da abundância total, por $55,9 \%$ de todos os exemplares encontrados nas lavouras e por $92,3 \%$ da abundância das espécies de sua subfamília. No entanto, todas as outras espécies e gêneros da subfamília Melanoplinae foram mais abundantes nos cerrados, com destaque para Propedies Hebard, 1931. A espécie O. punctata, a segunda mais abundante nesse estudo, contribuiu com $14,5 \%$ do efetivo total coletado, representando $22,9 \%$ dos exemplares obtidos nas lavouras, e 46,6\% de todas as espécies de Gomphocerinae (Tabela I).

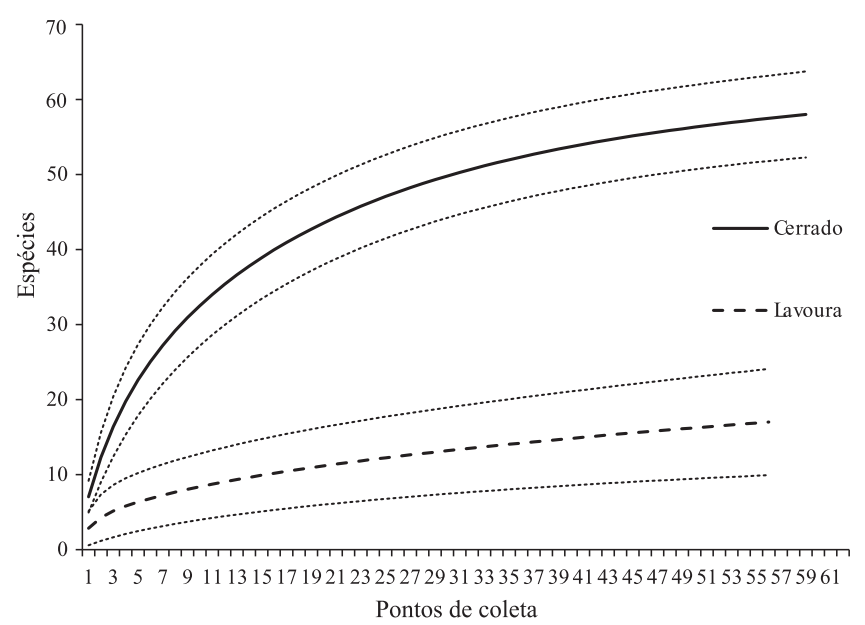

Fig. 3. Curva de acumulação (95\%) de espécies de gafanhotos em cerrados e lavouras na Chapada dos Parecis, Mato Grosso.

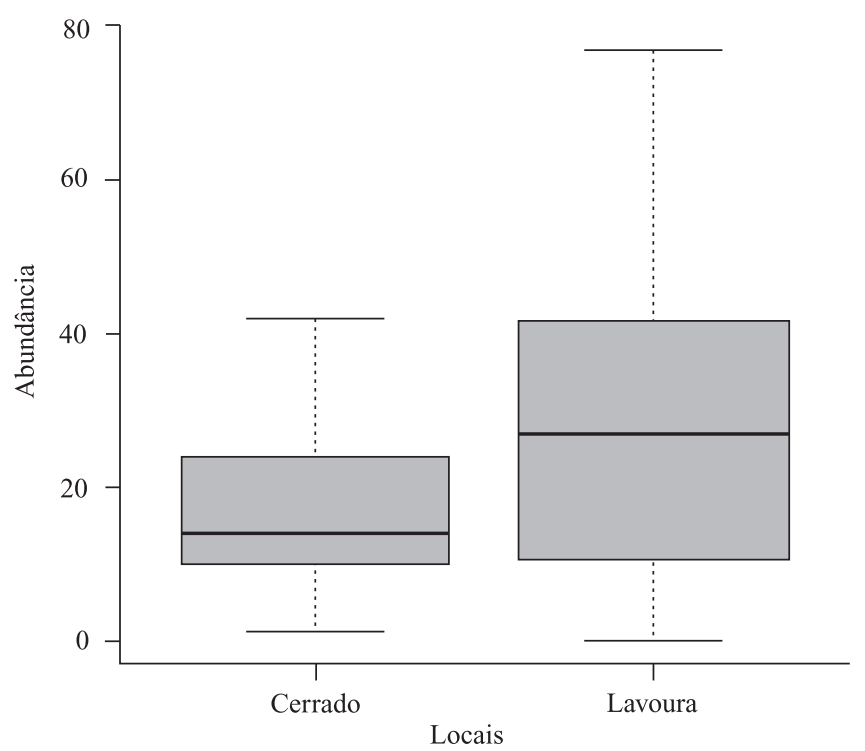

Fig. 4. Abundância de gafanhotos observada em 59 pontos nos cerrados (total $=1202$ indivíduos) e 56 nas lavouras (total $=1829$ indivíduos) da Chapada dos Parecis, Mato Grosso. A linha horizontal em negrito é a mediana, as caixas representam os quartis adjacentes à mediana e as hastes pontilhadas correspondem aos valores extremos. Áreas de lavouras comportam uma abundância significativamente maior que as áreas nativas $(\mathrm{p}=$ $0,035)$. 
Tabela I. Composição e abundancia de espécies de gafanhotos (Orthoptera: Acridoidea) entre ambientes de cerrado e lavoura na Chapada dos Parecis, Mato Grosso. R = espécies raras; I = espécies intermediárias; A = espécies de distribuição ampla.

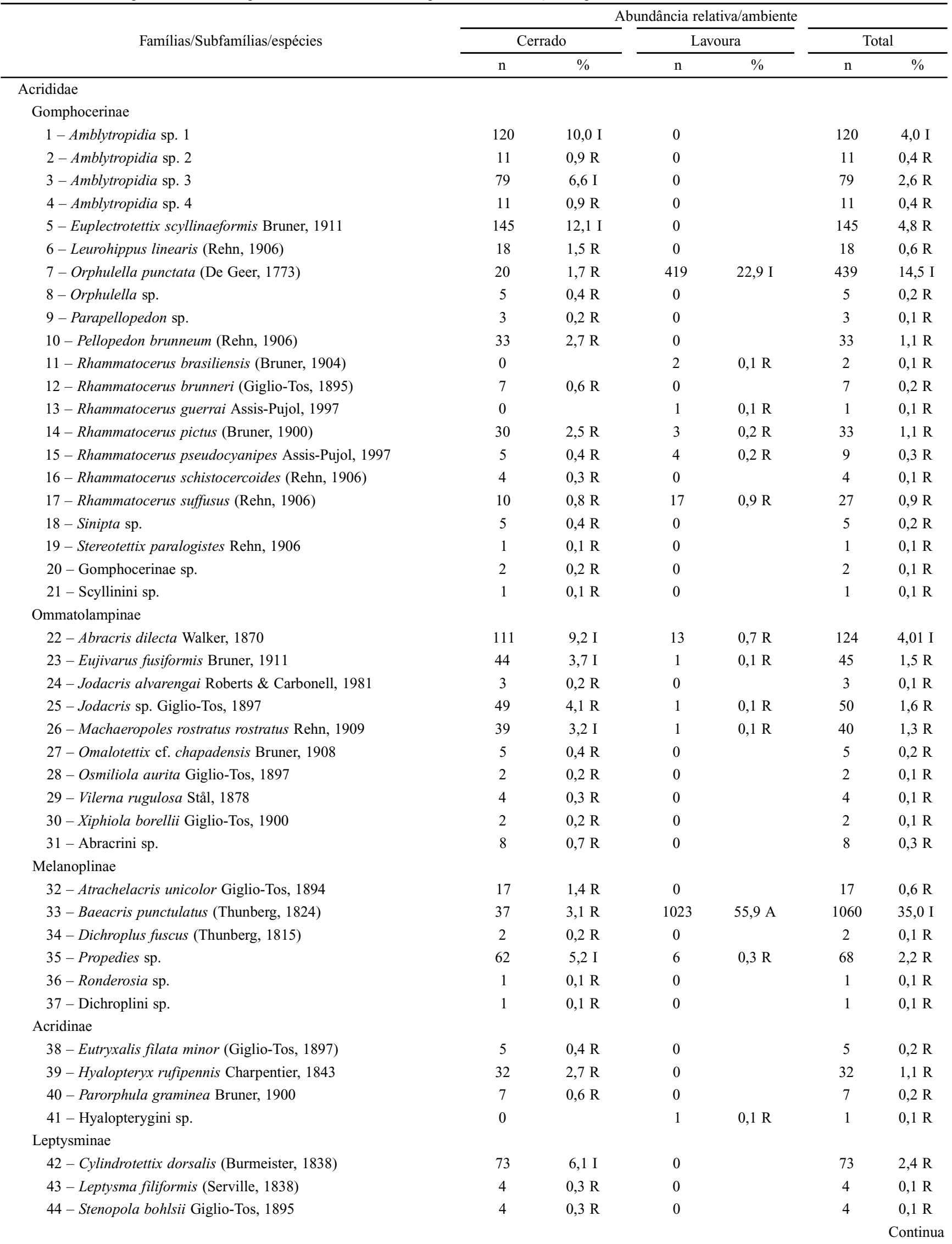




\begin{tabular}{|c|c|c|c|c|c|c|}
\hline \multirow{3}{*}{ Famílias/Subfamílias/espécies } & \multicolumn{6}{|c|}{ Abundância relativa/ambiente } \\
\hline & \multicolumn{2}{|c|}{ Cerrado } & \multicolumn{2}{|c|}{ Lavoura } & \multicolumn{2}{|c|}{ Total } \\
\hline & $\mathrm{n}$ & $\%$ & $\mathrm{n}$ & $\%$ & $\mathrm{n}$ & $\%$ \\
\hline \multicolumn{7}{|l|}{ Copiocerinae } \\
\hline 45 - Bucephalacris bohlsii (Giglio-Tos, 1898) & 59 & 4,9 I & 1 & $0,1 \mathrm{R}$ & 60 & $2,0 \mathrm{R}$ \\
\hline 46 - Chlorohippus roseipennis Bruner, 1911 & 11 & $0,9 \mathrm{R}$ & 0 & & 11 & $0,4 \mathrm{R}$ \\
\hline 47 - Zygoclistron trachystictum Rehn, 1905 & 6 & $0,5 \mathrm{R}$ & 0 & & 6 & $0,2 \mathrm{R}$ \\
\hline \multicolumn{7}{|l|}{ Proctolabinae } \\
\hline 48 - Eucephalacris borellii (Giglio-Tos, 1897) & 4 & $0,3 \mathrm{R}$ & 0 & & 4 & $0,1 \mathrm{R}$ \\
\hline \multicolumn{7}{|l|}{ Cyrtacanthacridinae } \\
\hline 49 - Schistocerca pallens (Thunberg, 1815) & 4 & $0,3 \mathrm{R}$ & 94 & $5,1 \mathrm{I}$ & 98 & $3,2 \mathrm{R}$ \\
\hline \multicolumn{7}{|l|}{ Romaleidae } \\
\hline \multicolumn{7}{|l|}{ Romaleinae } \\
\hline 50 - Abila bolivari Giglio-Tos, 1900 & 17 & $1,4 \mathrm{R}$ & 0 & & 17 & $0,6 \mathrm{R}$ \\
\hline 51 - Chromacris speciosa (Thunberg, 1824) & 21 & $1,87 \mathrm{R}$ & 0 & & 21 & $0,7 \mathrm{R}$ \\
\hline 52 - Chromacris nuptialis (Gerstaecker, 1873) & 10 & $0,8 \mathrm{R}$ & 0 & & 10 & $0,3 \mathrm{R}$ \\
\hline 53 - Staleochlora viridicata viridicata (Serville, 1838) & 1 & $0,1 \mathrm{R}$ & 0 & & 1 & $0,1 \mathrm{R}$ \\
\hline 54 - Titanacris picticrus marginalis Descamps \& Carbonell, 1985 & 1 & $0,1 \mathrm{R}$ & 0 & & 1 & $0,1 \mathrm{R}$ \\
\hline 55 - Tropidacris collaris (Stoll, 1813) & 2 & $0,2 \mathrm{R}$ & 0 & & 2 & $0,1 \mathrm{R}$ \\
\hline 56 - Xestotrachelus robustus (Bruner, 1911) & 1 & $0,1 \mathrm{R}$ & 0 & & 1 & $0,1 \mathrm{R}$ \\
\hline 57 - Xyleus gracilis (Bruner, 1905) & 9 & $0,7 \mathrm{R}$ & 0 & & 9 & $0,3 \mathrm{R}$ \\
\hline $58-$ Xyleus cf. lineatus (Bruner, 1906) & 13 & $1,1 \mathrm{R}$ & 0 & & 13 & $0,4 \mathrm{R}$ \\
\hline $59-$ Xyleus sp. & 6 & $0,5 \mathrm{R}$ & 0 & & 6 & $0,2 \mathrm{R}$ \\
\hline $60-$ Zoniopoda exilipes Bruner, 1906 & 1 & $0,1 \mathrm{R}$ & 0 & & 1 & $0,1 \mathrm{R}$ \\
\hline $61-$ Zoniopoda sp. 1 & 3 & $0,2 \mathrm{R}$ & 0 & & 3 & $0,1 \mathrm{R}$ \\
\hline $62-$ Zoniopoda sp. 2 & 3 & $0,2 \mathrm{R}$ & 0 & & 3 & $0,1 \mathrm{R}$ \\
\hline \multicolumn{7}{|l|}{ Ommexechidae } \\
\hline \multicolumn{7}{|l|}{ Ommexechinae } \\
\hline 63 - Descampsacris serrulatum (Thunberg, 1824) & 4 & $0,3 \mathrm{R}$ & 0 & & 4 & $0,1 \mathrm{R}$ \\
\hline 64 - Ommexecha virens Serville, 1831 & 4 & $0,3 \mathrm{R}$ & 242 & $13,2 \mathrm{I}$ & 246 & $8,1 \mathrm{I}$ \\
\hline Ninfas & 574 & $47,8 \mathrm{~A}$ & 976 & $53,4 \mathrm{~A}$ & 1550 & $51,1 \mathrm{~A}$ \\
\hline B. punctulatus & 5 & $0,9 \mathrm{R}$ & 242 & $24,8 \mathrm{I}$ & 247 & $15,9 \mathrm{I}$ \\
\hline Totais & 1202 & & 1829 & & 3031 & \\
\hline
\end{tabular}

As espécies com maior abundância nas lavouras são de ocorrência comum aos dois ambientes, no entanto com uma grande desproporção (Fig. 5A), destacando-se a B. punctulatus com $96,5 \%$ dos indivíduos capturados em ambientes de lavouras. Além desta espécie destacam-se outras três com mais de $90 \%$ dos indivíduos capturados nas lavouras: Orphulella punctata (95,4\%) Ommexecha virens Serville, 1831 (98,4\%) e Schistocerca pallens Thunberg, 1815 (95,9\%) (Tabela I).

A estrutura das taxocenoses encontradas nos cerrados e nas lavouras é demonstrada pela distribuição de abundância entre as espécies (Fig. 5). As espécies que mais contribuíram para a abundância significativamente maior nas lavouras em relação aos cerrados são: $B$. punctulatus $(\mathrm{H}=41,2 ; \mathrm{p}<$ $0,0001)$, O. punctata $(\mathrm{H}=17,2 ; \mathrm{p}<0,0001)$, S. pallens $(\mathrm{H}=$ $8,0 ; \mathrm{p}=0,0047)$ e $O$. virens $(\mathrm{H}=30,1 ; \mathrm{p}<0,0001)$. Nenhuma espécie teve ampla distribuição quando considerados todos os pontos de amostragem dentro da Chapada dos Parecis e apenas cinco são de distribuição intermediária, sendo que a maioria foi considerada rara no que se refere aos locais amostrados. No entanto, quando são considerados cada um dos ambientes inventariados, $B$. punctulatus é a única espécie de ampla distribuição nas áreas de lavouras. Nas áreas de cerrado, apesar de um maior número de espécies possuir distribuição intermediária, a maioria das espécies é considerada rara de acordo com o critério de Kemp (1992) (Tabela I).

\section{DISCUSSÃO}

É possível que seja deste trabalho o primeiro registro de ocorrência em Mato Grosso para Rhammatocerus pseudocyanipes, Sinipta sp., Eutryxalis filata minor, Parorphula graminea, Cylindrotettix dorsalis, Zygoclistron trachystictu, Chromacris nuptialis, Staleochlora viridicata viridicata e Xyleus gracilis. Outras cinco morfoespécies como Hyalopterygini sp., Scyllinini sp., Abracrini sp., Dichroplini sp. e Gomphocerinae sp. estão descritas na literatura em níveis taxonômicos de tribo ou subfamília, dentre as quais pode estar alguma espécie ainda sem registro. 

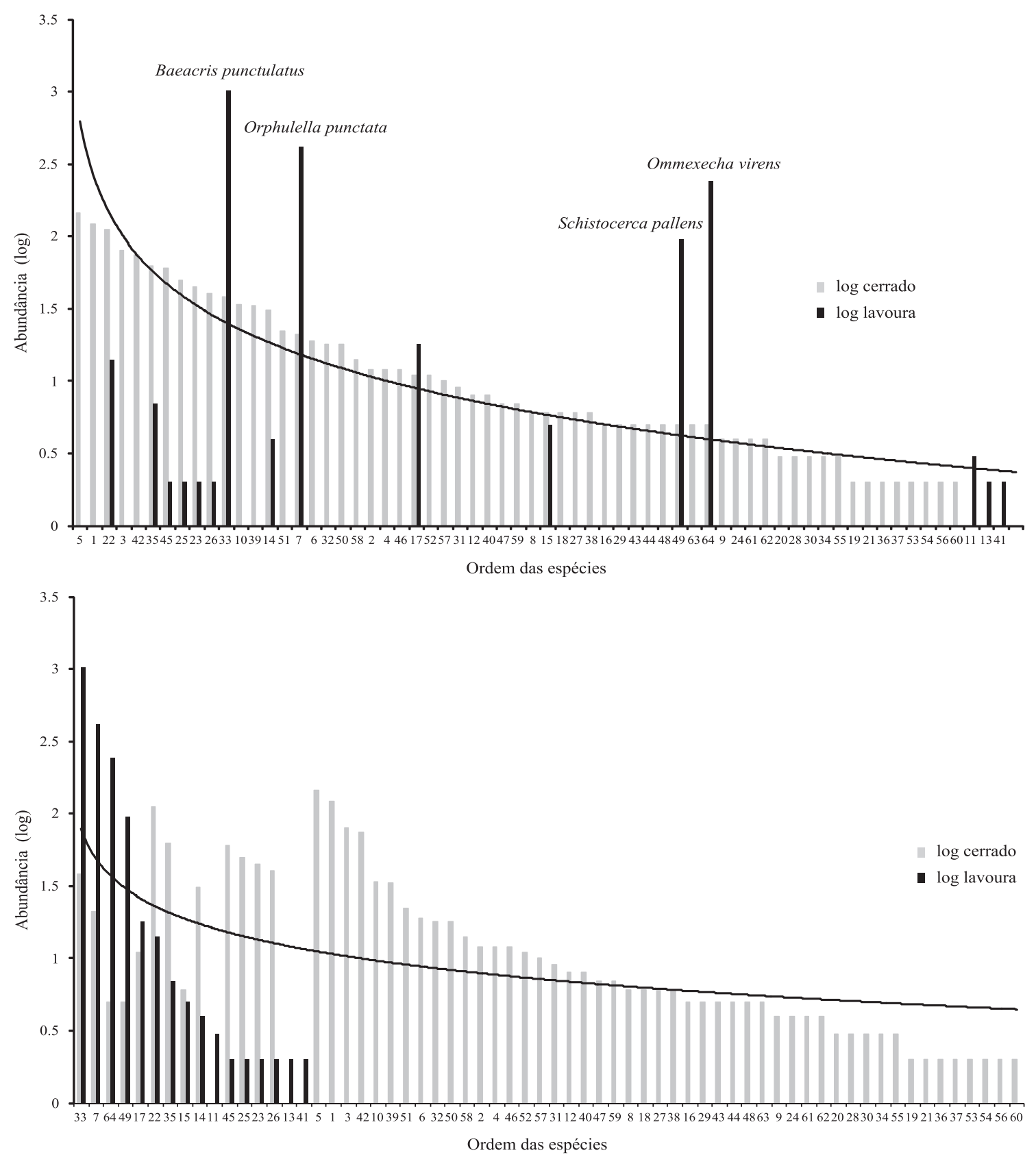

Fig. 5. Estrutura da taxocenose de gafanhotos na Chapada dos Parecis - MT. (A) Áreas de cerrados. (B) Áreas de lavouras. As abundâncias das espécies de cerrados estão representadas em barras cinzas e, em barras pretas, as de lavouras. Barras contíguas permitem uma comparação para as espécies comuns aos dois ambientes. Note-se o declive suave da estrutura de cerrados, indicando distribuição equitativa, ao contrário do que ocorre nas lavouras. Os números nas abscissas correspondem às espécies, conforme relacionadas na Tabela I.

As demais 57 espécies aqui registradas também o foram em levantamentos realizados desde o início do século XX e em diferentes regiões de Mato Grosso (Bruner 1911; Rehn 1906, 1909, 1913, 1918, 1944; Liebermann 1955; Ronderos 1976, 1977; Ronderos \& Sánchez 1983; Otte 1978; Descamps 1980, 1984; Roberts 1975; Roberts \& Carbonell 1979, 1980, 1981, 1982; Descamps \& Carbonell 1985; Amédégnato \& Poulain 1987; Assis-Pujol 1997; Carbonell 1969, 1986, 1995, 2002, 2004; Cigliano 2007). Tais estudos alcançaram 153 espécies de gafanhotos (Acridoidea). Entre as regiões ante- riormente investigadas, a mais visitada foi a Chapada dos Guimarães, uma unidade geomorfológica vizinha à Chapada dos Parecis, onde se registrou 105 espécies em 69 gêneros, dos quais 34 espécies e 22 gêneros correspondem aos encontrados na Chapada dos Parecis.

A semelhança entre as espécies catalogadas neste estudo, sobretudo nas áreas de cerrado, com aquelas obtidas em outros trabalhos sugere uma ampla área de distribuição das mesmas, as quais podem se estender por toda a área de cerrados do Brasil. Estes dados indicam que os cerrados e as 
lavouras da Chapada dos Parecis comportam pelo menos 37\% desta diversidade e se assemelha muito àquela da Chapada dos Guimarães. Considerando apenas as espécies encontradas neste levantamento pode-se inferir que as áreas nativas inventariadas contribuem com, no mínimo, 95\% da diversidade total de gafanhotos da Chapada dos Parecis $(\gamma=64$ espécies), enquanto que nas lavouras há apenas $25 \%$ da diversidade geral.

Foi observado o predomínio absoluto de espécies de Acrididae, dentre as quais diferentes espécies de Rhammatocerus Saussure, 1861, mas foram raros os exemplares de $R$. schistocercoides, principal espécie considerada praga da região em anos anteriores. De acordo com Miranda et al. (1996), o determinismo das explosões populacionais desta espécie precisa ser estudado em função da variabilidade das condições de precipitação durante a estação chuvosa e das queimadas durante a estação seca.

Dentro de Acrididae, a subfamília Gomphocerinae foi a mais rica e a que apresentou uma proporção mais equitativa na distribuição da abundância de indivíduos tanto em áreas antropizadas, quanto em áreas nativas, com quatro espécies presentes nos dois ambientes. É fato que esta proporção foi balanceada, sobretudo pela expressiva abundância de $O$. punctata nas lavouras, pela espécie Euplectrotettix scyllinaeformis Bruner, 1911 e gênero Amblytropidia Stal, 1873, presentes exclusivamente no cerrado. Capinera et al. (1997) e Squitier \& Capinera (2002) em levantamentos realizados em diferentes ambientes antropizados também relacionaram espécies dos gênero Amblytropidia e Orphulella dentre as mais abundantes. No entanto, ao contrário dos resultados aqui obtidos, ambas foram encontradas amplamente distribuídas entre e dentro dos ambientes, sugerindo que as espécies destes gêneros podem se adaptar a variadas condições ecológicas podendo se esperar que, assim como hoje ocorre com a Orphulella punctata, é possível que alguma espécie do gênero Amblytropidia possa colonizar as áreas de lavouras da Chapada dos Parecis.

Da mesma maneira quatro espécies de Ommatolampinae também estão presentes nos dois ambientes amostrados, mas diferentemente de Gomphocerinae, a maior abundância relativa foi encontrada nos cerrados, com destaque para Abracris dilecta Walker, 1870. De acordo com Roberts \& Carbonell (1981), esta espécie está associada a habitats arbustivos compatíveis com os ambientes de cerrado e em regiões de vegetação mais alta como as matas secas (Amédégnato \& Descamps 1980). Esta seletividade de habitat indica que esta espécie teria pouca capacidade de adaptação nas áreas agricultáveis, no entanto Sperber (1996) relacionou 14 espécies de plantas que são consumidas por $A$. dilecta a qual tem características biológicas de espécie generalista. Dentre estas se destacam algumas das famílias Leguminosae, Gramineae e Malvaceae que, por englobarem as principais espécies vegetais agrícolas cultivadas em Mato Grosso, tais como soja, arroz, milho e algodão podem ser fontes de recursos para que a espécie $A$. dilecta - assim como previsto para as Amblytropidia e já ocorre com B. punctulatus e $O$. punctata - com a possibilidade de se expandir pelas áreas agrícolas como uma resposta à ação antrópica.

A maior abundância relativa da subfamília Melanoplinae - a terceira mais rica de Acrididae - em relação à Gomphocerinae ocorreu em função de B. punctulatus. Em estudos objetivando comparar a riqueza de espécies e abundância relativa de gafanhotos entre áreas com comunidades de plantas nativas e exóticas nos pampas da Argentina, Torrusio et al. (2002) também relacionaram a subfamília Melanoplinae como a mais abundante. No entanto, ao contrário dos resultados do presente estudo, naquele caso Melanoplinae também foi a mais rica, seguida de Gomphocerinae.

A espécie B. punctulatus é considerada de grande distribuição geográfica e economicamente importante (Duranton et al. 1987; Bentos-Pereira 1989). O. punctata é citada como espécie que causa estragos menores, mas regulares e $O$. virens como espécie mal definida sob o aspecto de danos, mas cujos estragos seriam negligenciados (Duranton et al. 1987).

Dentre as espécies coletadas nas lavouras (16), além de B. punctulatus, pelo menos outras três (O. punctata, O. virens e $S$. pallens) são consideradas pragas agrícolas em algum cultivo (Silva et al. 1968; Duranton et al. 1987; Lecoq 1991) e foram as que notadamente influenciaram na maior abundância de gafanhotos nas lavouras (Fig. 5A). Quanto a $S$. pallens, praga de reconhecida importância no nordeste brasileiro (Duranton et al. 1987), apesar de ter sido detectada ocorrendo quase exclusivamente em lavouras, é encontrada de forma esparsa e até o presente momento não se caracterizou como de importância econômica para Mato Grosso. O mesmo ocorreu com $R$. schistocercoides e R. pictus, sendo a primeira muito conhecida nesse Estado, e a segunda citada dentre as grandes espécies devastadoras em outros países, considerada uma das 14 espécies mais prejudiciais da Argentina (Lange et al. 2005).

Apesar da ocorrência de três espécies somente em áreas de lavouras, não se pode afirmar que são exclusivas para esse ambiente, uma vez que no caso de Rhammatocerus guerrai Assis-Pujol, 1997, o primeiro exemplar foi originalmente coletado em ambiente de cerrado (Assis-Pujol 1997).

O manejo contínuo das áreas antropizadas, com sucessão ou rotação de cultivos, aliados à presença de plantas daninhas é o que pode estar favorecendo estas poucas espécies de gafanhotos na região de lavouras, conforme também diagnosticado por Marini et al. (2008). Em seu estudo, apenas algumas espécies de Caelifera foram capazes de desenvolver populações substanciais em áreas intensivamente manejadas. Estas espécies, exclusivas ou não, podem encontrar neste novo ambiente os recursos ideais para sua sobrevivência, em especial novas e nutritivamente ricas fontes alimentares, uma vez que as populações de gafanhotos, assim como de muitos outros insetos herbívoros, tendem a aumentar em função da qualidade das plantas (Joern 1996), ou mesmo apresentar maiores taxas de sobrevivência e longevidade quando o alimento é de melhor qualidade ou com maior disponibilidade, mesmo em presença de predador (Begon et al. 2006). 
As quatro espécies mais abundantes nas lavouras também são favorecidas pelas culturas de inverno tais como milho, milheto, girassol e crotalária onde é mínimo ou inexistente o uso de pesticidas. Com o início das chuvas a partir do mês de setembro, época que antecede o plantio da safra de verão, tendem a aumentar suas densidades populacionais ao ocuparem novos biótopos de reprodução, solos macios e mais favoráveis que aqueles encontrados em certos habitats dos cerrados, conforme sugeriu Lecoq (1991) ao estudar $R$. schistocercoides e Guerra et al. (2010) em relação ao $B$. punctulatus.

A significativa maior diversidade de gafanhotos no cerrado (61) em relação às áreas antropizadas por lavouras (16), pode ser resultado da influência da configuração do habitat sobre a taxocenose de Orthoptera. De acordo com Joern (2005), as características do habitat que maximizam a diversidade de gafanhotos incluem a heterogeneidade espacial dos habitats com estrutura aberta, a existência de várias espécies vegetais como fonte de alimento, estrutura heterogênea para os predadores, e espaços que propiciem a termorregulação, além de plantas com alto valor nutritivo, compatível com os cerrados. Pode-se sugerir que a maior riqueza de espécies de gafanhotos nas áreas de cerrados é resultado da estrutura da vegetação uma vez que a diversidade de artrópodes tende a incrementar com o aumento desta estrutura, padrão consistente com a teoria ecológica (Pianka 1966).

Pelo mesmo raciocínio, a simplificação do ambiente que caracteriza as áreas de cultivo responderia pela menor diversidade encontrada na área antropizada. O baixo número de espécies de gafanhotos nas lavouras, se comparado com o encontrado nos cerrados, indica uma baixa capacidade de adaptação da maioria das espécies aos ambientes antropizados. Esses resultados corroboram com os estudos de diferentes cenários de uso da terra, nos quais o número médio de espécies de gafanhotos diminuiu com as alterações ambientais, sobretudo com o uso agrícola (Steck et al. 2007; Marini et al. 2008). Os constantes níveis de perturbação devido aos manejos nestes ambientes, acentuadamente com o uso de pesticidas pelos agricultores durante a safra de verão visando outras pragas, assim como a redução dos recursos alimentares em função da substituição dos cerrados pelas plantas cultivadas e sua ausência na entressafra, também explicam esta menor diversidade de espécies. Da mesma forma, a prática da fertilização ao tempo em que incrementa a produtividade do cultivo principal, resulta em um concomitante decréscimo da diversidade de espécies vegetais (Gough et al. 2000; Begon et al. 2006). O uso de fertilizantes favorece determinadas espécies vegetais em detrimento de outras, diminuindo a riqueza por causa da exclusão competitiva interespecífica homogeneizando ainda mais a paisagem antropizada, com reflexo negativo na diversidade de artrópodes. Além disso, a alta taxa de uso dos solos nas áreas agricultáveis no início das chuvas, período mais adequado à reprodução dos insetos em geral, também pode levar a reduções populacionais significativas de determinadas espécies de gafanhotos nestes ambientes face à destruição de sítios de oviposição. De acordo com Knapp et al. (1998), se os distúrbios são freqüentes no espaço e no tempo, muitas espécies são eliminadas e, por outro lado, se os distúrbios são raros, a exclusão competitiva reduz a diversidade.

A distribuição ranqueada das abundâncias das espécies de gafanhotos mostra que as taxocenoses de gafanhotos nas áreas de cerrados e de lavouras não apresentam estruturas similares (Fig. 5), revelando padrões dentro das expectativas da teoria ecológica (Ricklefs \& Miller 2000; Magurran 2004; Begon et al. 2006), em que ambientes nativos, de estrutura mais complexa, respondem por um maior número de espécies com distribuição mais equilibrada em relação aos ambientes antropizados.

Nos ambientes antropizados, tanto a composição vegetacional simplificada quanto a alta taxa de perturbação diminuem o número de espécies, que por sua vez apresentam desproporcional divisão do nicho espacial. Nestas situações são conhecidos os efeitos negativos da homogeneização das paisagens e da agricultura intensiva sobre a biodiversidade (Benton et al. 2003). Nas lavouras há poucas espécies respondendo por quase toda a abundância, e a estrutura se aproxima daquela preconizada pelo modelo série geométrica (Fig. 5B). Whittaker (1972) e Tokeshi (1993) confirmam que tal modelo é característico de uma taxocenose pobre em espécies, com alta dominância, pequena harmonia ou pequena equitabilidade, típico de comunidades em estágios recentes de sucessão. Báldi \& Kisbenedek (1997) encontraram padrões muito semelhantes aos registrados nas lavouras durante o presente estudo, obtendo altas densidades de gafanhotos e baixa riqueza em ambientes alterados se comparados com ambientes naturais. Torrusio et al. (2002) também encontraram significantes incrementos na densidade de uma espécie de Melanoplinae (Dichroplus elongatus Giglio-Tos, 1894) em ambientes perturbados pelo uso de pastagens em relação a áreas de vegetação nativas, compatível com o registrado neste trabalho para B. punctulatus.

Já na área de cerrados, o maior número de espécies distribui-se de maneira mais equitativa que sua contraparte antropizada, e a estrutura daquela taxocenose assemelha-se ao padrão de distribuição log-série (Fisher et al. 1943) (Fig. $5 \mathrm{~A}$ ), onde cada espécie idealmente ocupa uma fração constante do nicho espacial restante. Sugihara (1980) explicou que neste modelo o conjunto de espécies divide o recurso disponível (ou nicho espacial) paulatina e sequencialmente desde as espécies dominantes até as mais raras, produzindo um declive mais equilibrado da curva de distribuição das espécies e refletindo a maior complexidade da composição de espécies na área nativa (Tokeshi 1993).

Os resultados sugerem que as áreas hoje ocupadas por lavouras perderam pelo menos 47 das 61 espécies que ali existiam quando aquele ambiente era cerrado e hoje servem de habitat preferencial para quatro espécies (Fig. 5A) que também habitam o ambiente natural. Estes registros atuais são indicativos de que tais espécies estão sendo favorecidas pela ação do homem e podem se tornar importantes pragas agrícolas à região. Esta situação é alarmante para o agrone- 
gócio da região, e deve ser tomada com especial atenção pelos órgãos públicos de defesa fitossanitária. Ainda mais alarmante é considerar que a perda de diversidade aqui registrada para os Acridoidea, ao se substituir o ambiente nativo pelo antropizado, também está ocorrendo com muitos outros taxa dos quais sequer temos registro.

Este foi o primeiro passo para o conhecimento da composição de um importante grupo de insetos que possui inúmeras espécies consideradas pragas aos sistemas agrícolas. No entanto, apesar da ocorrência de algumas delas na Chapada dos Parecis, nem todas foram encontradas associadas a danos às lavouras. É possível que algumas ocorrências não estejam sendo avaliadas ou até mesmo negligenciadas com vistas a possíveis perdas, pois o sistema de produção considera a praga-chave na adoção de medidas de controle, com os gafanhotos sendo indiretamente controlados. Em face desta possibilidade, é necessário que estudos sejam feitos no sentido de se avaliar o potencial destas espécies de gafanhotos que já tem registros como pragas em outras regiões para se determinar se são ou poderão se tornar pragas para os cultivos da região.

\section{AGRADECIMENTO}

Os autores agradecem aos estudantes Andréia Cristina Tavares de Mello (UFMT), Everton Luis Silva Costa (UNEMAT) e Silvana Silva Amaral (UNIVAG) pela valiosa ajuda nas coletas em campo e a João Alves de Lima Filho pela contribuição na análise dos dados. Especial agradecimento à Maria Marta Cigliano, Miguel Ângelo Monné, Christiane Amédégnato (in memorian), Maria Kátia Matiotti da Costa e Cristiane Vieira de Assis Pujol pela ajuda na identificação do material e ao Michel Lecoq pelas sugestões na metodologia de coleta. Este estudo foi custeado pelo Ministério da Agricultura, Pecuária e Abastecimento (MAPA/SFA/MT).

\section{REFERENCIAS}

Amédégnato, C. 1974. Les genres d'acridiens néotropicaux, leur classification par familles, sous familles et tribus. Acrida 3: 193-204.

Amédégnato, C. \& M. Descamps. 1980. Évolution des populations d'orthoptères d'amazonie du nord-ouest dans les cultures traditionnelles et les formations secondaires d'origine anthropique. Acrida 9: 1-33.

Amédégnato, C. \& S. Poulain. 1987. Les acridiens néotropicaux. I: Proctolabinae amazoniens (Orthoptera: Acridoidea). Annales de la Société Entomologique de France 23: 399-434.

Assis-Pujol, C. V. 1997. Duas novas espécies brasileiras de Rhammatocerus Saussure, 1861 (Acrididae, Gomphocerinae, Scyllinini). Boletim do Museu Nacional, nova série Zoologia 380: 1-10.

Báldi, A. \& T. Kisbenedek. 1997. Orthopteran assemblages as indicators of grassland naturalness in Hungary. Agriculture Ecosystems and Environment 66: 121-129.

Begon, M.; C. R. Townsed \& J. L. Harper. 2006. Ecology: from individuals to ecosystems. $2^{\text {nd }}$ Ed., Oxford Blackwell xii +738 p.

Benton, T. G.; J. A. Vickery \& J. D. Wilson. 2003. Farmland biodiversity: is habitat heterogeneity the key? Trends in Ecology and Evolution 18: 182-188.

Bentos-Pereira, A. 1989. Distribución geográfica de las especies del género Dichroplus Stal (Orthoptera, Acrididae, Melanoplinae). Revista Bra- sileira de Entomologia 33: 31-47.

Brasil, Ministério das Minas e Energia, M. M. E. 1982. Folha SD-21 Cuiabá. Levantamento de recursos naturais. Vol. 26, Secretaria Geral, Projeto RADAM-BRASIL, 544 p.

Bruner, L. 1911. South American Acridoidea. Annals of the Carnegie Museum 8: 5-147.

Capinera, J. L.; C. W. Scherer \& J. B. Simkins. 1997. Habitat associations of grasshoppers at the Macarthur Agro-Ecology Research Center, Lake Placid, Florida. Florida Entomologist 80: 253-261.

Carbonell, C. S. 1969. Revision of the genus Zygoclistron Rehn, 1905 (Orthoptera: Acridoidea). Transactions of the American Entomological Society 95: 571-602.

Carbonell, C. S. 1986. Revision of the Neotropical Genus Tropidacris (Orthoptera, Acridoidea, Romaleidae, Romaleinae). Proceedings of the Academy of Natural Sciences of Philadelphia 138: 366-402.

Carbonell, C. S. 1988. Rhammatocerus schistocercoides (Rehn, 1906) especie prejudicial para la agricultura en la region centro oeste de Brasil (Orthoptera, Acrididae, Gomphocerinae). Boletim do Museu Nacional, Série Zoologia 318: 1-17.

Carbonell, C. S. 1995. Revision of the tribe Scyllinini, Nov. (Acrididae: Gomphocerinae), with descriptions of new genera and species. Transactions of the American Entomological Society 121: 87-152

Carbonell, C. S. 2002. The grasshopper tribe Phaeopariini (Acridoidea: Romaleidae). Publications on Orthopteran diversity. Philadelphia, The Orthopterists' Society, 148 p.

Carbonell, C. S. 2004. The genus Xyleus Gistel 1848 (Acridoidea, Romaleidae, Romaleinae). Journal of Orthoptera Research 13: 63-133.

Cigliano, M. M. 2007. Review of the South American genus Eurotettix Bruner (Orthoptera, Acridoidea, Melanoplinae). Systematic Entomology 32: 176-195.

Constantino, R. 2005. Padrões de diversidade e endemismo de térmitas no bioma cerrado, p. 319-333. In: A. O. Scariot; J. C. S. Silva \& J. M. Felfili (eds.). Biodiversidade, ecologia e conservação do cerrado. Brasília, Ministério do Meio Ambiente, 439 p.

Colwell, R. K. 2006. EstimateS: Statistical estimation of species Richness and shared species from samples. Version 8.2.0. Disponível em: http:/ /viceroy.eeb.uconn.edu/estimates (acessado em 29 de agosto de 2010).

Descamps, M. 1980. La faune dendrophile néotropicale V[a]. Seconde revue des Proctolabinae amazoniens et guyanais (Orthoptères, Acrididae). Annales de la Société Entomologique de France 16: 19-47

Descamps, M. 1984. Revue préliminaire de la tribu des Copiocerini (Orth. Acrididae). Mémoires du Muséum National d'Histoire Naturelle Nouvelle série. Série A, Zoologie 130: 1-72.

Descamps, M. \& C. S. Carbonell. 1985. Revision of the Neotropical Arboreal Genus Titanacris (Orthoptera, Acridoidea, Romaleidae). Annales de la Société Entomologique de France 21: 259-285.

Duranton, J. F.; M. Launois; M. H. Launois-Luong \& M. Lecoq. 1982. Manuel de prospection acridienne en zone tropicale sèche. Paris, Cirad-Gerdat Tome 2, 723-1487.

Duranton, J. F.; M. Launois; M. H. Launois-Luong \& M. Lecoq. 1987. Guia prático de luta contra os gafanhotos devastadores no Brasil. Montpellier, France: Fao, CIRAD/PRIFAS, 161 p.

Eades, D. C.; D. Otte; M. M. Cigliano \& H. Braun. 2009. Orthoptera Species File Online. Version 2.0/4.0. Disponível em: http:// orthoptera.speciesfile.org. (acessado outubro de 2010).

Fisher, R. A.; A. S. Corbet \& C. B. Williams. 1943. The relation between the number of species and the number of individuals in a random sample of an animal population. Journal of Animal Ecology 12: 42-58.

Gangwere, S. K.; M. C. Muralirangan \& M. Muralirangan. 1997. The Binomics of Grasshoppers, Katydids and Their Kin. Wallingford, CAB International, xiii +529 p.

Gebeyehu, S. \& M. J. Samways. 2002. Grasshopper assemblage response to a restored national park (Mountain Zebra National Park, South Africa). Biodiversity and Conservation 11: 283-304.

Gotelli, N. J. \& R. K. Colwell. 2001. Quantifying biodiversity: procedures and pitfalls in the measurement and comparison of species richness. Ecology Letters 4: 379-391. 
Gough, L.; C. W. Osenberg; K. L. Gross \& S. L. Collins. 2000. Fertilization effects on species density and primary productivity in herbaceous plant communities. Oikos 89: 428-439.

Guerra, W. D. 2001. Rhammatocerus schistocercoides (Rehn, 1906) y otros acridoideos de importância econômica em Brasil, p. 19-32. In: I Curso Internacional sobre Ecologia, manejo y control de langosta voladora. Ciudad Victoria, Dinámica Impresa, 232 p.

Guerra, W. D.; P. C. Oliveira, \& L. Barrientos-Lozano. 2010. Life history and population dynamics of Baeacris punctulatus (Thunberg, 1824) (Orthoptera: Acrididae) in the state of Mato Grosso, Brazil. Journal of Orthoptera Research 19: 333-340.

Hammer, O.; D. A. T. Harper \& P. D. Ryan. 2001. PAST: Paleontological Statistics Software Package for Education and Data Analysis. Palaeontologia Electronica 4 (1): 9p. Disponível em: http://palaeo-electronica.org/2009_2/past/ issue1_01.htm (acessado em 13 de setembro de 2009).

Joern, A. 1996. Host plant quality and grasshopper populations. In: Cunningham G L, Sampson M W (Eds.) Grasshopper Integrated Pest Management User Handbook. Washington, United States Department of Agriculture, Animal and Plant Health Inspection Service, Technical Bulletin 1809, p. IV.4-1-IV.4-6.

Joern, A. 2005. Distrubance by fire frequency and bison grazing modulate grasshopper assemblages in tallgrass praire. Ecology 86: 861-873.

Kemp, W. P. 1992. Temporal variation in rangeland grasshopper (Orthoptera: Acrididae) communities in the steppe region of Montana, USA. Canadian Entomologist 124: 437-450.

Knapp, A. K.; J. M. Briggs; D. C. Hartnett \& S. L. Collins (Eds). 1998. Grassland dynamics: long-term ecological research in tallgrass prairie. Oxford, Oxford University Press, 386 p.

Lange, C. E.; M. M. Cigliano \& M. L. de Wysiecki. 2005. Los acridoideos (Orthoptera: Acridoidea) de importancia económica en la Argentina. In: $2^{\circ}$ Curso: Manejo Integrado da Langosta Centroamericana (Schistocerca piceifrons piceifrons, Walker) y Acridoideos Plaga en America Latina. Ciudad Victoria, Dinámica Impresa, Memorias 302 p.

Lecoq, M. 1991. Gafanhotos do Brasil. Natureza do Problema e Bibliografia. Montpellier, France: Embrapa/Nma, Cirad/Prifas, 157 p.

Liebermann, J. 1955. Primeira relação sistemática dos acridoideos do Brasil. Memórias do Instituto Oswaldo Cruz 53: 329-344.

Magurran, A. E. 2004. Measuring Biological Diversity. Oxford, Blackwell Publishing Company $256 \mathrm{p}$.

Marini, L.; P. Fontana; M. Scotton \& S. Klimek. 2008. Vascular plant and Orthoptera diversity in relation to grassland management and landscape composition in the European Alps. Journal of Applied Ecology 45: 361-370.

Miranda, E. E.; M. Lecoq; I. Pierozzi Jr.; J. F. Duranton \& M. Batistella. 1996. O gafanhoto do Mato Grosso, Balanço e perspectivas de 4 anos de pesquisas, 1992-1996. Relatório Final do Projeto "Meio Ambiente e Gafanhotos Pragas no Brasil. Campinas EMBRAPA-NMA, CIRAD-GERDAT-PRIFAS $146 \mathrm{p}$

Mittermeier, R. A.; N. Myers; P. R. Gil \& C. Mittermeier. 1999. Hotspots: earth's biologically richest and most endangered terrestrial ecoregions. Mexico City, Cemex/Conservation International, $420 \mathrm{p}$.

Oksanen, J.; R. Kindt; P. Legendre; B. O’Hara; G. L Simpson; P. Solymos; M. H. H. Stevens \& H. Wagner. 2009. Vegan Community Ecology Package version 1.15-4. Disponível em: http://cc.oulu.fi/jarioksa (acessado em 14 de agosto de 2010)

Otte, D. 1978. Revision of the grasshoppers tribe Orphulellini (Acrididae: Gomphocerinae). Proceedings of the Academy of Natural Sciences of Philadelphia 131: 52-88.

Pianka, E. R. 1966. Latitudinal gradients in species diversity: a review of concepts. The American Naturalist 100: $33-46$.

R Development Core Team. 2009. R: A language and environment for statistical computing. R Foundation for Statistical Computing, Vienna, Austria. Disponível em: http://www.R-project.org (acessado em 26 de novembro de 2009)

Rehn, J. A. G. 1906. Notes on South American grasshoppers of the subfamily Acridinae (Acrididae) with descriptions of new genera and species. Proceedings of the United States National Museum 30: 371-396.
Rehn, J. A. G. 1909. On Brazilian grasshoppers of the subfamilies Pyrgomorphinae and Locustinae (Acridinae of authors). Proceedings of the United States National Museum 36: 109-163.

Rehn, J. A. G. 1913. A contribution to the knowledge of the Orthoptera of Argentina. Proceedings of the United States National Museum 65: 273-379.

Rehn, J. A. G. 1918. Descriptions of one genus and fifteen news species of tropical American Orthoptera. Trans. American Orthoptera. Transactions of the American Entomological Society 44: 321-371.

Rehn, J. A. G. 1944. A revision of the locusts of the group Hyalopteryges (Orthoptera; Acrididae; Acridinae). Transactions of the American Entomological Society 70: 181-234.

Ribeiro, J. F. \& B. M. T. Walter. 1998. Fitofisionomias do Bioma Cerrado, p. 87-166. In: S. M. Sano \& S. P. D. Almeida (Eds.). Cerrado: ambiente e flora. Brasília, EMBRAPA $556 \mathrm{p}$.

Ricklefs, R. E. \& G. L. Miller. 2000. Ecology. Fourth edition. New York, W. E. Freeman and Company, 896 p.

Roberts, H. R. 1975. A revision of the genus Cylindrotettix including new species (Orthoptera: Acrididae: Leptysminae). Proceedings of the Academy of Natural Sciences of Philadelphia 127: 29-43.

Roberts, H. R. \& C. S. Carbonell. 1979. A revision of the genera Stenopola and Cornops (Orthoptera, Acrididae, Leptysminae. Proceedings of the Academy of Natural Sciences of Philadelphia 131: 104-130.

Roberts, H. R. \& C. S. Carbonell. 1980. Concluding revision of the subfamily Leptysminae (Orthoptera, Acrididae). Proceedings of the Academy of Natural Sciences of Philadelphia 132: 64-85.

Roberts, H.R. \& C. S. Carbonell. 1981. A revision of the neotropical genus Abracris and related genera (Orthoptera, Acrididae, Ommatolampinae). Proceedings of the Academy of Natural Sciences of Philadelphia 133: $1-14$.

Roberts, H. R. \& C. S. Carbonell. 1982. A revision of the grasshopper genera Chromacris and Xestotrachelus (Orthoptera, Romaleidae, Romaleinae). Proceedings of the California Academy of Natural Sciences 43: 43-58.

Ronderos, R. A. 1976. Revisión del género Parascopas Bruner (Orthoptera: Acrididae, Melanoplianae). Revista de la Sociedad Entomológica Argentina 35: 175-192.

Ronderos, R. A. 1977. Notas para una revisión de la subfamilia Ommexechinae VIII. El género Ommexecha Serville (Orthoptera: Acridiomorpha). Revista de la Sociedad Entomológica Argentina 36: 97111.

Ronderos, R. A. \& N. E. Sánchez. 1983. Revision del genero Propedies Hébard (Orthoptera, Acridiae, Melanoplinae). Revista de la Sociedad Entomológica Argentina 42: 171-218.

Rosas-Costa, J. A. 1966. Preparación de Acridoidea y Tettigonioidea. Geotrópica. 12: 110-112.

Santos, F. M.; C. A. L. Carvalho \& R. F. Silva. 2004. Diversidade de abelhas (Hymenoptera: Apoidea) em uma área de transição Cerrado-Amazônia. Acta Amazônica 34: 319-328

Silva, A. G. A.; C. R. Gonçalves; D. M. Galvão; A. J. L. Gonçalves; J. Gomes; M. N. Silva \& L. Simoni. 1968. Quarto Catálogo dos Insetos que Vivem nas Plantas do Brasil. Seus Parasitos e Predadores. Parte II $-1^{\circ}$ Tomo. Rio de Janeiro, Ministério da Agricultura, $622 \mathrm{p}$.

Soares, S. A.; W. F. Antonialli-Junior \& S. E. Lima-Junior. 2010. Diversidade de formigas epigéicas (Hymenoptera, Formicidae) em dois ambientes no Centro-Oeste do Brasil. Revista Brasileira de Entomologia 54: 76-81.

Sperber, C.F. 1996. Field diet of the grasshopper Abracris dilecta Walker (Orthoptera, Acrididae). Revista Brasileira de Zoologia 13: 127-135.

Squitier, J. M. \& J. L. Capinera. 2002. Habitat associations of Florida grasshoppers (Orthoptera: Acrididae). Florida Entomologist 85: 235-244.

Steck, C. E.; M. Bürgi; J. Bolliger; F. Kienast; A. Lehmann \& Y. Gonseth. 2007. Conservation of grasshopper diversity in a changing environment. Biological Conservation 138: 360-370.

Sugihara, G. 1980. Minimal community structure: an explanation of species abundance patterns. The American Naturalist 116: 770-787.

Tokeshi, M. 1993. Species abundance patterns and community structure. 
Advances in Ecological Research 24: 111-186.

Torrusio, S.; M. M. Cigliano \& M. L. de Wysiecki. 2002. Grasshopper (Orthoptera: Acridoidea) and plant community relation ships in the Argentine Pampas. Journal of Biogeography 29: 221-229.

Whittaker, R. H. 1972. Evolution and measurement of species diversity. Taxon 21: 213-251.
Wysiecki, M. L.; M. M. Ciglinao \& S. Torrusio. 2005. Ecologia y Dinâmica de Saltamontes (Orthoptera: Acridoidea) de las Pampas, Argentina, p. 80-91. In: $2^{\circ}$ Curso Internacional: Manejo Integrado da Langosta Centroamericana (Schistocerca piceifrons piceifrons, Walker) y Acridoideos Plaga en America Latina. Ciudad Victoria, Dinámica Impresa, $302 \mathrm{p}$. 